\title{
SOME ASPECTS OF THE COUNTING PROPERTIES OF DIAMOND
}

\author{
by G. P. FREEMAN and H. A. VAN DER VELDEN
}

Physisch Laboratorium der Rijks Universiteit te Utrecht, Nederland

\begin{abstract}
Synopsis
Experiments carried out on diamond crystal counters are described. The use of a new diamond specimen leads to the same value for the product of the mobility and the mean free time in the crystal as had been found for a previous specimen, the data for which have been already published by the same authors.

The energy necessary for the liberation of one electron in a diamond crystal appears to be $8,6 \mathrm{e} . \mathrm{V}$. or less. Evidence is given that the parts of a mixed crystal used for the counting of $\alpha$-particles are of type II (transparent to ultra violet light).
\end{abstract}

$\S 1$ Introduction. For a crystal showing electron-conductivity under irradiation with particles or quanta, it is possible to define several characteristic quantities; the mobility $v$ of the electrons in the crystal, the mean time $T$ that a liberated electron stays in the conduction band and the energy $E$ necessary to liberate an electron. The behaviour of the crystal under the particular radiation can be described with the aid of these quantities. Under the simple circumstances of one single kind of electron trap and if a homogenous distribution of these traps in the crystal is considered, these quantities will be sufficient to account for the properties of the crystal. However, a description of the experimental behaviour of real crystals is rather difficult because in general these simple circumstances are not present: very little is known about the depths of the traps and the distribution of the traps in depth and in space. It is usually assumed that:

1) The electrons remain trapped for either a long time or a very short time when compared with the time of measurement;

2) The traps are homogeneously distributed in the crystal. It has already been shown ${ }^{1}$ ) (this paper is afterwards referred to as paper I) 
that at least for diamond, the second assumption is not usually correct. This was done by measuring different effects at different spots of one diamond, under identical external circumstances (same external fields, some irradiation with $\alpha$-particles and red light). A consequence of this is that another result might be obtained if another diamond is used, or if the irradiation is performed with $\alpha$ - or $\beta$ particles, electrons of low energy, or quanta. This is especially true if only the effect produced when the entire crystal is irradiated with, for example, light is measured. Thus, structure-dependant results may be expected. To solve this difficulty a diamond used as a crystal counter was irradiated with $\alpha$-particles and the induced conductivity of each particle was measured separately. As in paper I, only part of the effects were compared under varying circumstances, namely the part consisting of the largest effects only"

A second problem is introduced by the creation of a space charge during the measurements. This means that the result will depend upon the history of the crystal. Up till now the best elimination of space charge in diamond is probably given by K. G. $M\left(\mathrm{~K} \text { a y }{ }^{2}\right)^{3}$ ), whose recently improved experiments give consistent results for one diamond specimen (irradiation with electrons of low energy). As it was intended to measure the individual effect caused by $a$-particles the method of $\mathrm{M} \mathrm{c} \mathrm{K} \mathrm{a} \mathrm{y}$ is less convenient for our purpose. Therefore, another method was studied, namely a simultaneous irradiation with red or infrared light during the experiments ${ }^{4}$ ). This enables the determination of Hecht's function, which gives the relation between the magnitude of the signal and the field strength in the crystal, and in consequence thereof, determines a minimum value for the product of the mobility $"$ and the meantime $T$.

This paper contains a repetition of the previously described experiments with another diamond specimen and under different circumstances; at the same time the absolute number of electrons liberated is measured. This number, together with the energy of the $\alpha$-particles (of Polonium), determines the energy $E$ necessary to take one electron into the conduction band. The values of $E$ found for different circumstances happened to be different and the reason for this discrepancy is given. As before, in determining the energy $E$, only the largest signals were considered. This method has the advantage - above those by which one irradiates a larger surface obtaining an average result - of eliminating the influence of internal cracks, 
laminated structure and inhomogenity in the distribution of the electron traps. The importance of this elimination was discussed in paper I, and we have already utilised the laminated structure of some diamonds to explain observed phenomena ${ }^{4}$ ).

Up till now, it is not yet known which part of each diamond will count $a$-particles and which will not ${ }^{5}{ }^{6}{ }^{6}$ ) ${ }^{7}$ ). In general it is known that two different kinds of diamond ${ }^{8}$ ) exist distinguished principally $\left.{ }^{9}\right)$ by the property that diamond I absorbs in the ultraviolet (2000-3000 $\AA$ ) while diamond II transmits in this region. As many counting crystals are partly transparent, it is difficult to decide whether both parts have counting properties or, if not, which part is counting. In the third section of this paper the experimental data is given which shows clearly that the ultraviolet-transparent parts (diamond II) are the most important - if not the only - parts contributing in the counting of $\alpha$-particles.

The experimental arrangement was the same as that described in the two previous papers ${ }^{1}{ }^{4}$ ).

$\S 2$. Hecht's function and the energy per liberated electron. In order to determine the liberation energy per electron, Hecht's function was measured, the absolute number of electrons liberated by one $\alpha$-particle verified, and then the energies of the $\alpha$-particles at the moment of entrance into the crystal compared. Hecht's function gives the relation between the charge pulse $Q$ measured and the field strength in the crystal:

$$
\delta=\Omega\left(1-e^{\left.-\frac{1}{\Omega}\right)}\right.
$$

Here $\delta=x / l=Q / N e$. ( $N$ is the number of liberated electrons, $X$ the average distance covered in the crystal by the electrons, $l$ the thickness of the crystal) and $\Omega=F v T / l$ ( $F$ is the field strength, $v$ the mobility, $T$ the average free time of an electron). For any particular diamond specimen, the method of determining this function has already been explained in paper I. $\mathrm{Ne}$ is found, in arbitrary measure, by comparing the experimental values with the theoretical curve. The calibration was obtained by inducing a known charge on the grid of the first valve of the amplifier. The energy of the $\alpha$-particles at the moment of entering the crystal was calculated from the distance between the Polonium-source and the crystal. Under normal circumstances this energy was $4,0 \mathrm{MeV}$. 
Using this calibration for the diamond for which Hecht's function in the stationary situation and with red light was given in paper $I$, a charge pulse for an $\alpha$-particle of $2,15.10^{5}$ e.c. was obtained; this means an energy of $18,6 \mathrm{eV}$ per liberated electron. This value is much larger than one should expect. According to $S$ e i t $z^{10}$ ) from theory a value between 7 and $10 \mathrm{eV}$ might be expected. A. J. A h e a $\mathrm{n}^{5}$ ) found a minimum value of $10 \mathrm{eV}$ using $a$-particles and $\mathrm{K}$. $\mathrm{G}$.

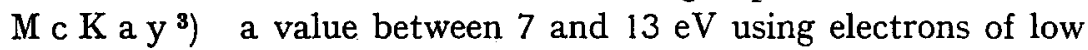
energy.

There are two reasons why the value obtained in our investigations might be too large, firstly it might be that even the largest charge pulses do not cover an average distance of more than 0,3 times the thickness of the crystal and secondly that a number of electrons might be trapped before making a contribution to the signal. As a consequence of the very good correlation between the experimental data and the theoretical curve (see paper 1) the first possibility can be eliminated. The long time this crystal has been in use also suggests the second possibility may be true. Moreover, after the measurements have been interrupted, the pulses obtained of resuming measuring were for a short time larger than usual. For that reason the crystal was not used for a week. After a week, (with $F=6000$ $\mathrm{V} / \mathrm{cm}$ ) - in absence of red light - pulses were obtained for a few minutes corresponding with $4,5 \cdot 10^{5}$ electron charges. This number was derived assuming that the curve of $\mathrm{H} \mathrm{e} \mathrm{ch} \mathrm{t}$ did not depend on the rate of recombination. The corresponding energy-value wanted is $8,9 \mathrm{eV}$. One has to distinguish between a higher trapping chance in a region that has been under irradiation for a longer period, as supposed here, and a normal recombination chance. The higher trapping chance depends on the history of the crystal; the recombination is independant of this history.

Further investigation is therefore necessary to elucidate the question: "Does the trapping depend on the field strength?" for if this is true than the value of $v T$ already found is incorrect. The answer is, that a possible dependence of the rate of recombination on the field strength may be eliminated as a consequence of the correspondence between the experimental data and the theoretical function.

To investigate this Hecht's function was redetermined, this time with a new, completely unused diamond specimen. To eliminate a 
possible influence of red light, and to diminish the influence of earlier measurements, a counting time of 15 minutes was used for each value and the crystal was not used for one day between every two values determined. At $60 \mathrm{~V}$ and $100 \mathrm{~V}$ only a small amount of red light was used while above $100 \mathrm{~V}$ experiments were carried out in the absence of red light. The results are given in fig. 1. It appears that within the limits of accuracy the curve gives the same value of $5,3 \cdot 10^{-5} \mathrm{~cm}^{2} /$ Volt for $v T$ as in the case of the other diamond specimen with red light and long time measurements (paper I). Consequently no important influence of circumstances is found.

When calibrated, the energy per liberated electron appeared to be even less than the value of $8,9 \mathrm{eV}$ which had been found previously. This indicates that trapping, if present, is less. The number of electron charges at $700 \mathrm{~V}$ was $4.10^{5}$, corresponding to an energy of 8,6 $\mathrm{eV}$ per electron. The accuracy is about $7 \%$. Because we do not

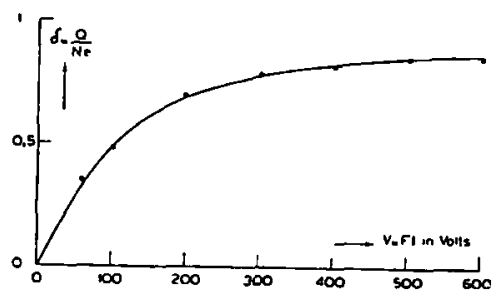

Fig. 1. know the influence of recombination, it is possible that the real energy value is smaller.

One phenomenon occurred which we did not understand. During the first days the crystal gave scarely any effect for field strengths below $1500 \mathrm{~V} / \mathrm{cm}$; above this value the signal grew rapidly with tension. After some days of use the counting of the crystal improved and larger signals were produced. Moreover the charge pulse increased during the first minutes of every measurement after which it decreased again, probably owing to the growing space charge.

\$3. The counting sections of diamond. In order to explain some experiments ${ }^{4}$ ) it has already been supposed that a counting diamond possesses a laminated structure. The size of the counting cells however was unknown. This, together with contradiction in literature, makes it desirable to investigate the characteristic properties of counting diamonds more closely.

Friedman, $\mathrm{Birks}$ and $\mathrm{Gauvin}{ }^{9}$ ) were the first authors who pointed out that a possible correlation exists between the counting properties and the type of diamond. They found the best $\gamma$-rays-counting diamonds to be of type II, ultraviolet transpa- 
rent ones. Shortly afterwards A. J. A h e a r $\mathrm{n}^{5}$ ) with $\alpha$-particles obtained the largest signals in crystals classified as type I. However he did not exclude the possibility that his crystals were mixed ones of which only the type I parts counted. Then R. H of $s t a d t e r^{6}$ ) supposed the type I to have a mosaic structure and type II a laminated one. In consequence the type II would give the electrons the possibility of a longer free path in one direction and thus constitute the better kind of counting crystals. This idea however was severely contradicted by $\mathrm{K}$. L o n s d a l e ${ }^{7}$ ) who declared the type I crystal to be nearly the most perfect crystal known. That type II shows a laminated structure was demonstrated by the experiments of $\mathrm{Kr}$ is h m a n and $\mathrm{R}$ a m a c hand ran, published in a symposium under direction of $\operatorname{Sir} C . V . R$ a $m$ a ${ }^{8}{ }^{8}$ ). They investigated the crystals optically as well as by means of $x$-rays.

Our experience is that no type I crystal counts $a$-particles; only those which are at least partly transparent might show an induced conductivity by $\alpha$ - and $\beta$-particles. Moreover the supposition of a mosaic or laminated structure is needed to explain the behaviour of a counting crystal $\left.{ }^{4}\right)$. In addition there is still another consideration, given below, which for counting diamonds indicates at least a laminated structure. It is possible to give a minimum for the average linear dimension of the hypothetical cells. For the diamond described in paper I - as well as for the other investigated by us - the magnitude of the maximum signals decreased after about ten minutes. This implies that on the average in every counting cell at least one $\alpha$-particle is trapped at this moment.

If the experimental data given are considered (about $6.10^{2} \alpha$ particles per minute, e.c. $6.10^{3}$ in 10 minutes, an efficiency of $25 \%$, a surface of $6 \mathrm{~mm}^{2}$ ), one obtains for a linear dimension $x$ :

$$
\bar{x}^{2}>\left(1,5 \cdot 10^{6} / 6 \cdot 10^{3}\right) \mu^{2} \text {, so } \bar{x}>15 \mu \text {. }
$$

A maximum value might also be given for the same linear-dimension of the cells. The distribution of the magnitude of the signals given by mono-energetical $\alpha$-particles has been measured and such a distribution is sketched in fig. 2 . With a resolution power determined by the noise of the amplifier we find about seventeen maxima. If we explain these maxima in the way we believe to be correct by introducing one cell (at least one) for each maximum, this gives a maximum value 
for the square of the linear dimension of such a cell $\bar{x}^{2}<\left(1,5.10^{6} / 17\right) \mu^{2}$ which means, $\bar{x}<300 \mu$.

Moreover in the third direction, the depth, a similar value is found. The charge is trapped.after having covered a distance varying between 100 and $1000 \mu$. This too suggests a mosaic or laminated structure: If one compares these values $15 \mu<X<300 \mu$ with those given by $\mathrm{Ram} \mathrm{ach}$ a ndran for the dimension of the laminated cells in diamond II which lies between 10 and $100 \mu$, it must be concluded that the agreement between the results is very good, the more so, since our estimate is a rough one.

In our opinion these experimental data and considerations result in the conclusion that the ultraviolet transparent parts, those of diamond II, are the most likely to count, at least for $a$-particles. The experimental

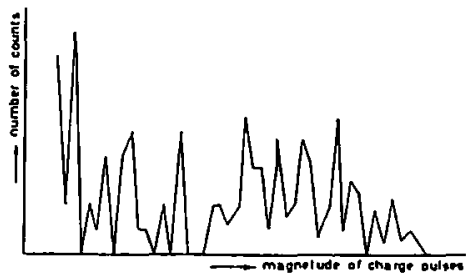

Fig. 2. contradiction with A. J. Ahearn's results is not conclusive, as his crystal might be a mixed one, of which a small part of type II counts.

$\S 4$. Conclusion. We have already discussed some striking difference with older experiments. K. G. M c K a y with his recently improved method ${ }^{3}$ ) obtained an average value of $v T=8,3 \cdot 10^{-6} \mathrm{~cm}^{2}$ / Volt with one diamond specimen and for a varying irradiation. We are convinced that the value of $v T=5,3 \cdot 10^{-5} \mathrm{~cm}^{2} /$ Volt we found is more correct, especially because the same value was obtained with two different specimens. Moreover, in our experiments the influence on the result caused by the inhomogenous distribution of traps in different parts of the crystals, and the influence of internal cracks was eliminated. To get the same result as K.G. Mc K a y, it is only necessary to average the distributions of $\delta=Q / N e$ as given in fig. 2 e.q. For $F=6000 \mathrm{~V} / \mathrm{cm}$, as in this figure, the average value must be $2 / 5$ of the maximum value in order to have a near correspondence with the value found by $\mathrm{K}$. G. M c K a y. It is evident that our average values lie very near to those of $K$. G. M $\mathrm{CK}$ a $y$, thus proving again the correctness of the deductions.

If a trapping cross section $\sigma$ of $10^{-15} \mathrm{~cm}^{2}$ is assumed, one finds the number of traps $N$ in the best parts of the crystal:

$$
N=1 / T \sigma \mu
$$


to be about $2.10^{15} / \mathrm{cm}^{3}$ (where $u$ is the thermal velocity, the mobility of the electrons is supposed to be $900 \mathrm{~cm}^{2} / \mathrm{V} \mathrm{sec}$ according to $\mathrm{Klick}$ and $\mathrm{Maurer}{ }^{10}$ )).

The energy necessary to liberate an electron appeared to be 8,6 $\mathrm{eV}$, which is a little less than the average value of $10 \mathrm{eV}$ found by $\mathrm{Ahearn}$ and also by $\mathrm{McK}$ a y. The question remains if our value is also too high, as one does not know the influence of recombination.

Finally it should be pointed out that the influence of recombination and trapping appears to be more important in investigations of this kind than one would expect according to older publications. More attention must be paid to the influence of the method on the result than has been done up till now. This is especially important if a crystal is used continually for a long period.

We wish to express our thanks and appreciation to Professor J. M. W. Mil a $\mathrm{z}$ for his kind and helpful interest and his enlightening discussions of our investigation. Also to $\mathrm{J}$. $\mathrm{H}$ ove $\mathrm{s} \mathrm{t} \mathrm{r}$ e y d t who assisted in these experiments, and to the "Stichting voor Fundamenteel Onderzoek der Materie" which under assistance of the „,Stichting voor Zuiver Wetenschappelijk Onderzoek" made this investigation possible.

Received 23-1-51.

\section{REFERENCES}

1) Freeman, G. P., and van der Velde n, H. A., Physica 16 (1950) 486.

2) M c K a y, K. G., Phys. Rev. 74 (1948) 1606.

3) M c K a y, K. G., Phys. Rev. 77 (1950) 816.

4) Van der Velde n, H. A., and Free m a n, G. P., Physica 16 (1950) 493.

5) A he a r n, A. J., Phys. Rev. 73 (1948) 1113.

6) H of st a d ter, R., Phys. Rev. 73 (1948) 631.

7) L o nsda l e, K., Phys. Rev. 73 (1948) 1467.

8) $\mathrm{R}$ a m a $\mathrm{n}$ et al, Proc. Ind. Acad. Sci. Symp. 1, 19A, 1944; Symp. II, 24A, 1946. K ris h m a n, R. S., Symp. I, A 19 (1944) 298; R a m a c h a nd r a n, G. M., Symp I, A 10 (1944) 304.

9) Friedman, Birks and Gauvin, Phys. Rev. 73 (1948) 186.

10) Se it z, F., Phys. Rev. 76 (1949) 1376. 